\title{
Real world adverse events of interspinous spacers using Manufacturer and User Facility Device Experience data
}

Received December 2, 2020

Revised February 28, 2021

Accepted March 2, 2021

\section{Corresponding author}

Nitish Aggarwal, M.D., MBA

Department of Anesthesiology, Yale

New Haven Hospital, 20 York St., New

Haven, CT, USA

Tel: 1-4752109716

Fax: 1-2037856664

E-mail: nitish.aggarwal@yale.edu

\section{Nitish Aggarwal and Robert Chow}

Department of Anesthesiology, Yale New Haven Hospital, New Haven, CT, USA
Background: Lumbar spinal stenosis is a condition of progressive neurogenic claudication that can be managed with lumbar decompression surgery or less invasive interspinous process devices after failed conservative therapy. Popular interspinous process spacers include X-Stop, Vertiflex and Coflex, with X-Stop being taken off market due to its adverse events profile.

Methods: A disproportionality analysis was conducted to determine whether a statistically significant signal exists in the three interspinous spacers and the reported adverse events using the Manufacturer and User Facility Device Experience (MAUDE) database maintained by the US Food and Drug Administration.

Results: Statistically significant signals were found with each of the three interspinous spacer devices (Coflex, Vertiflex, and X-Stop) and each of the following adverse events: fracture, migration, and pain/worsening symptoms.

Conclusions: Further studies such as randomized controlled trials are needed to validate the findings.

Keywords: Coflex; Compression fracture; Disproportionality analysis; Interspinous spacer; Interventional; MAUDE database; Veriflex; X-Stop.

\section{INTRODUCTION}

Lumbar spinal stenosis is a condition of progressive neurogenic claudication that is usually worsened by lumbar extension and relieved by lumbar flexion. Initial management of lumbar spinal stenosis consists of decreasing stress on lower back and physical therapy. However, the conservative treatments are often ineffective and surgical treatment is then considered.

The underlying issue with surgical management is that many of the patients with lumbar spinal stenosis are elderly with multiple comorbidities. This increases the risk complications associated with general anesthesia and delayed recovery after the procedure. Some complications include screw misplacement, unintended durotomy (cerebrospinal fluid [CSF] leak), infection, deep venous thrombosis and postoperative anemia [1]. These concerns led to development of minimally invasive lumbar decompression and interspinous process devices. The potential advantages of these minimally invasive procedures over spine surgery include shorter procedure time, ability to perform the procedure under local anesthesia, minimal blood loss, and less risk of CSF leak [2]. Three of the most common interspinous devices include Coflex (Paradigm Spine LLC, USA), Vertiflex (Boston Scientific, USA) and X-Stop (Medtronic Inc., USA).

This is an Open Access article distributed under the terms of the Creative Commons Attribution Non-Commercial License (http://creativecommons.org/licenses/by-nc/4.0) which permits unrestricted non-commercial use, distribution, and reproduction in any medium, provided the original work is properly cited.

Copyright (c) the Korean Society of Anesthesiologists, 2021 
Because these devices are relatively new and widespread adoption in clinical practice has only recently started to occur, serious adverse events can arise that were not discovered previously in the early trials of the device. As such, the purpose of this study is to use disproportionality analysis to determine whether statistically significant signals exist with each of the three interspinous spacers/plates and each of the following serious adverse events: allergic reaction, cerebrospinal fluid leak, spinous process or transverse process fracture, infection, malfunction of the device, migration of the device, and pain/worsening symptoms.

\section{MATERIALS AND METHODS}

\section{MAUDE database}

The Manufacturer and User Facility Device Experience (MAUDE) is a database that houses medical device reports for suspected device-associated deaths, serious injuries and malfunctions that are submitted to the Food and Drug Administration (FDA), the USA. These medical device reports can be submitted by mandatory reporters (manufacturers, importers, and device user facilities) or by voluntary reporters such as health care professionals, patients and consumers [3].

A pharmacovigilance analysis was performed on this database for three interspinous spacers/plates (Coflex, Vertiflex, and X-Stop) and the following adverse events: allergic reaction, CSF leak, fracture, infection, malfunction, migration and pain/ worsening symptoms. Approval by an institutional review board or human subjects' committee was not required for this analysis as it was performed on retrospective public domain safety data.

\section{Data processing}

Reports were downloaded ranging from January 1, 2010 to July 31, 2020 and stratified by the product class "Prosthesis, Spinous Process Spacer/Plate." In total, over 8 million medical device reports were analyzed and over 500 reports were found in the above product class during the specified time range. Based on the event description for each report, duplicates were identified and removed. In addition, some medical device reports contained event descriptions from abstracts/papers. Since it could not be verified whether these adverse event reports were also included as a separate medical device reports, these reports were excluded from the analysis to prevent double counting. A graphical depiction of data acquisition and processing is provided in Fig. 1.

\section{Disproportionality analysis}

A disproportionality analysis allows for quantification of the relationship by examining the expected count of device-adverse event reports to the actual count. Some measurements in a disproportionality analysis using a frequentist approach include proportional reporting ratio (PRR), relative reporting ratio, reporting odds ratio (ROR), and chi-squared with Yates' correction. A Bayesian approach for disproportionality analysis includes the measurement information component (IC) [4].

Three criterion can be used independently to determine if a device-adverse event combination is a statistically significant signal:

1. Lower limit of the $95 \%$ confidence interval for ROR greater than one [5]

2. PRR is greater than or equal to 2 , chi-squared is greater than or equal to 4 , and the number of device/drug to adverse event reports greater than or equal to 3 , a statistically significant signal is found [6]

3. Lower limit of the $95 \%$ confidence interval for IC $\left(\mathrm{IC}_{025}\right)$ greater than [7]

ROR, PRR, chi-squared with Yates' correction and $\mathrm{IC}_{025}$

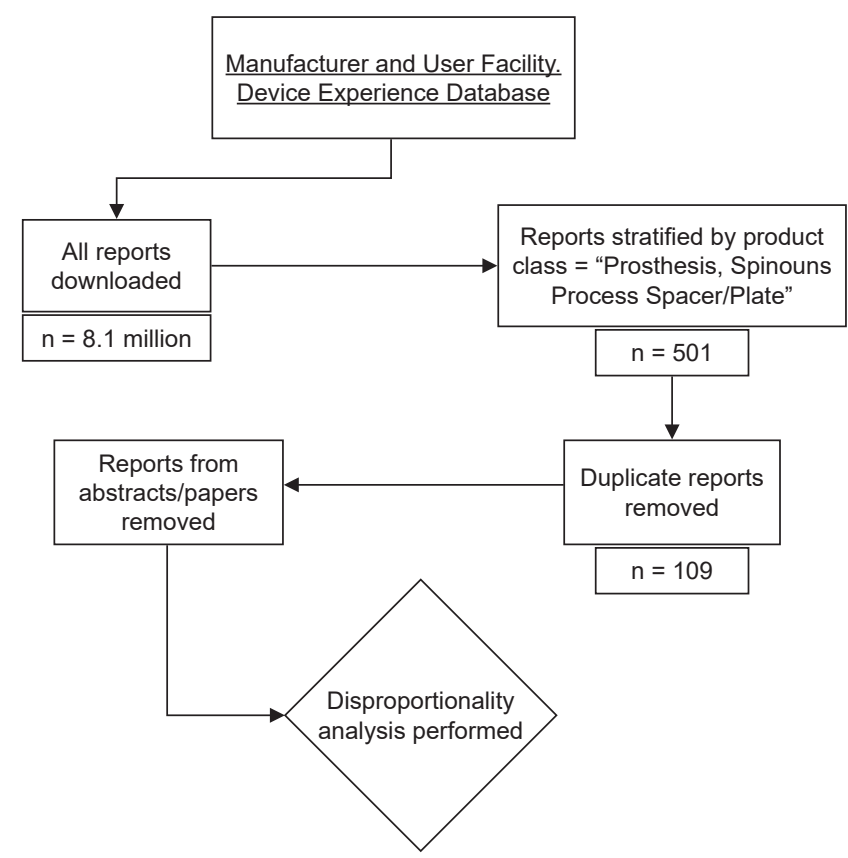

Fig. 1. Graphical depiction of data acquisition and processing. 
are calculated in this analysis for each combination of the spinous process spacers/plates and adverse events. Formulas to calculate PRR and ROR are shown in Fig. 2 and formulas for chi-square with Yates' correction and $\mathrm{IC}_{025}$ have previously been described [8-10].

\section{RESULTS}

The total number of adverse event reports for each of the devices were: Coflex $=39$; Vertiflex $=108$; X-Stop $=245$. Measures from the disproportionality analysis for each of the device-adverse event combinations are shown in Table 1. Using the criterion of ROR Lower 95\% CI greater than 1, a statistically significant signal is found for Coflex and (CSF leak, fracture, infection, migration, and pain/worsening symptoms); for Vertiflex and (allergic, fracture, migration, and pain/worsening symptoms); for X-Stop and (allergic, CSF leak, fracture, migration, and pain/worsening symptoms) (Table 2). Using the criteria consisting of PRR greater than or equal to 2 and chi-squared greater than or equal to 4 and the number of device/ drug to adverse event reports greater than or equal to 3 , a significant signal is found for Coflex and (fracture, infection, migration, and pain/worsening symptoms); for Vertiflex and (allergic, fracture, migration, and pain/worsening symptoms); for X-Stop and (fracture, migration, and pain/worsening symptoms] (Table 2).

A heat map is displayed in Fig. 3 that contains the $\mathrm{IC}_{025}$ values for each device and adverse event combination. The color of each of the boxes is based on the $\mathrm{IC}_{025}$ value with values greater than 0 being green and values less than 0 being yellow/red. In addition, each box is sized relative to each of the other boxes based upon $\mathrm{N}$ (the number of medical device reports for that specific device and adverse event combination). This heat map can be used as a quick reference for which a device and adverse event combination contained a significant signal. The $\mathrm{IC}_{025}$ can only be used to determine whether a statistically significant signal is found or not. A higher or lower $\mathrm{IC}_{025}$ does not signify a more or less statistically significant signal. As such, the heat map cannot be used to compare $\mathrm{IC}_{025}$ of one device and its adverse events with $\mathrm{IC}_{025}$ of other devices and their adverse events. Using the criterion of a $\mathrm{IC}_{025}$ greater than zero, a significant signal is found for Coflex and (fracture, migration, and pain/worsening symptoms); for Vertiflex and (fracture, migration, and pain/worsening symptoms); for X-Stop and (fracture, migration, and pain/worsening symptoms).

\section{DISCUSSION}

Using all three disproportionality criteria, a statistically significant signal was found for each of the interspinous spacer devices (Coflex, Vertiflex, and X-Stop) and the adverse events of fracture, migration, and pain/worsening symptoms. Reviewing the literature on Coflex, five studies mentioned spinous process fractures in total of 35 patients [11]. In addition, several studies mentioned malposition of

\begin{tabular}{|l|l|l|l|}
\hline & Device of interest & All other devices & All devices \\
\hline Adverse event of interest & A & B & A+B \\
\hline All other adverse events & C & D & C+D \\
\hline All adverse events & A+C & B+D & $A+B+C+D$ \\
\hline
\end{tabular}

$$
\begin{gathered}
\text { Proportional Reporting Ratio }(P R R)=\frac{\frac{A}{\frac{A+C}{B}}}{B+D} \\
\text { PRR Standard Deviation }(S D)=\sqrt{\frac{C}{A *(A+C)}+\frac{D}{B *(B+D)}} \\
\text { PRR Confidence Interval }=e^{\ln (P R R \pm 1.96 * S D)} \\
\text { Reporting Odds Ratio }(R O R)=(A / C) /(B / D) \\
\text { ROR Standard Deviation }(S D)=\sqrt{\frac{1}{A}+\frac{1}{B}+\frac{1}{C}+\frac{1}{D}} \\
\text { ROR Confidence Interval }=e^{\ln (R O R \pm 1.96 * S D)}
\end{gathered}
$$

Fig. 2. Formulas for calculating PRR and ROR. 
Table 1. The Count and Disproportionality Measures for Each of the Spinous Process Spacers/plates and Adverse Event Combinations in the MAUDE Database from January 1, 2010 to July 31, 2020

\begin{tabular}{|c|c|c|c|c|}
\hline Adverse events & Count (n) & ROR $(95 \% \mathrm{Cl})$ & PRR (95\% Cl) & Chi-squared with Yates correction \\
\hline \multicolumn{5}{|l|}{ Allergic } \\
\hline Coflex & 0 & - & - & - \\
\hline Vertiflex & 3 & $9.15(2.90-28.82)^{*}$ & $8.92(2.92-27.23)^{\dagger}$ & 13.96 \\
\hline X-Stop & 3 & $3.97(1.27-12.39)^{*}$ & $3.93(1.28-12.11)$ & 3.97 \\
\hline \multicolumn{5}{|l|}{ CSF leak } \\
\hline Coflex & 1 & $60.09(8.25-437.77)^{*}$ & $58.57(8.46-405.56)$ & 13.66 \\
\hline Vertiflex & 0 & - & - & - \\
\hline X-Stop & 2 & $18.80(4.67-75.62)^{*}$ & $18.65(4.69-74.19)$ & 18.09 \\
\hline \multicolumn{5}{|l|}{ Fracture } \\
\hline Coflex & 8 & $10.18(4.68-22.16)^{*}$ & $8.30(4.47-15.40)^{\dagger}$ & 45.45 \\
\hline Vertiflex & 15 & $6.37(3.69-10.98)^{*}$ & $5.62(3.51-8.99)^{\dagger}$ & 53.77 \\
\hline X-Stop & 46 & $9.12(6.62-12.57)^{*}$ & $7.60(5.86-9.86)^{\dagger}$ & 263.49 \\
\hline \multicolumn{5}{|l|}{ Infection } \\
\hline Coflex & 5 & $3.61(1.41-9.22)^{*}$ & $3.27(1.44-7.42)^{\dagger}$ & 6.01 \\
\hline Vertiflex & 7 & $1.70(0.79-3.66)$ & $1.65(0.81-3.39)$ & 1.26 \\
\hline X-Stop & 14 & $1.49(0.87-2.55)$ & $1.46(0.88-2.43)$ & 1.65 \\
\hline \multicolumn{5}{|l|}{ Malfunction } \\
\hline Coflex & 3 & $0.05(0.01-0.15)$ & $0.12(0.04-0.35)$ & 53.62 \\
\hline Vertiflex & 11 & $0.06(0.03-0.11)$ & $0.16(0.09-0.27)$ & 139.74 \\
\hline X-Stop & 17 & $0.04(0.02-0.07)$ & $0.11(0.07-0.17)$ & 359.26 \\
\hline \multicolumn{5}{|l|}{ Migration } \\
\hline Coflex & 8 & $20.83(9.57-45.32)^{*}$ & $16.76(9.04-31.09)^{\dagger}$ & 104.61 \\
\hline Vertiflex & 23 & $21.84(13.78-34.63)^{*}$ & $17.40(12.11-25.01)^{\dagger}$ & 343.54 \\
\hline X-Stop & 36 & $13.91(9.76-19.81)^{*}$ & $12.01(8.88-16.24)^{\dagger}$ & 356.67 \\
\hline \multicolumn{5}{|c|}{ Pain/worsening symptoms } \\
\hline Coflex & 13 & $4.78(2.46-9.30)^{*}$ & $3.52(2.26-5.49)^{\dagger}$ & 23.19 \\
\hline Vertiflex & 27 & $3.19(2.06-4.93)^{*}$ & $2.64(1.90-3.66)^{\dagger}$ & 28.59 \\
\hline X-Stop & 94 & $5.95(4.60-7.70)^{*}$ & $4.05(3.46-4.75)^{\dagger}$ & 235.27 \\
\hline
\end{tabular}

MAUDE: Manufacturer and User Facility Device Experience, CSF: cerebrospinal fluid, Cl: confidence interval, ROR: reporting odds ratio, PRR: proportional reporting ratio. Coflex (Paradigm Spine LLC, USA). Vertiflex (Boston Scientific, USA). X-Stop (Medtronic Inc., USA). *Statistically significant signals as determined by ROR Lower $95 \% \mathrm{Cl}$ greater than $1 .{ }^{\dagger}$ Statistically significant signals as determined by PRR greater than or equal to 2 and chi-squared greater than or equal to 4 and the number of device/drug to adverse event reports greater than or equal to 3 . The ${ }^{\dagger}$ is only placed next to the PRR value.

Table 2. Statistically Significant Adverse Events for Three Spinous Process Spacers/plates from the MAUDE Database (January 1, 2010 to July 31 , 2020) Using Three Disproportionality Analysis

\begin{tabular}{|c|c|c|c|c|c|c|c|}
\hline \multirow{2}{*}{ Device } & \multirow{2}{*}{ Significant signal criteria } & \multicolumn{6}{|c|}{ Adverse event } \\
\hline & & Allergic & CSF leak & Fracture & Infection & Migration & Pain/worsening symptoms \\
\hline \multirow{3}{*}{ Coflex } & Criterion 1 & & $\mathrm{x}$ & $x$ & $x$ & $x$ & $x$ \\
\hline & Criterion 2 & & & $x$ & $x$ & $x$ & $x$ \\
\hline & Criterion 3 & & & $x$ & & $x$ & $x$ \\
\hline \multirow[t]{3}{*}{ Vertiflex } & Criterion 1 & $x$ & & $x$ & & $x$ & $x$ \\
\hline & Criterion 2 & $x$ & & $x$ & & $x$ & $x$ \\
\hline & Criterion 3 & & & $x$ & & $x$ & $x$ \\
\hline \multirow[t]{3}{*}{ X-Stop } & Criterion 1 & $x$ & $x$ & $x$ & & $x$ & $x$ \\
\hline & Criterion 2 & & & $x$ & & $x$ & $x$ \\
\hline & Criterion 3 & & & $x$ & & $x$ & $x$ \\
\hline
\end{tabular}

MAUDE: Manufacturer and User Facility Device Experience, CSF: cerebrospinal fluid, Cl: confidence interval, ROR: reporting odds ratio, PRR: proportional reporting ratio, IC: information component. Coflex (Paradigm Spine LLC, USA). Vertiflex (Boston Scientific, USA). X-Stop (Medtronic Inc., USA). Criterion 1: ROR Lower 95\% Cl greater than 1. Criterion 2: PRR greater than or equal to 2 and chi-squared greater than or equal to 4 and the number of device/drug to adverse event reports greater than or equal to 3. Criterion 3: $\mathrm{IC}_{025}$ greater than zero. 


\begin{tabular}{|c|c|}
\hline \multicolumn{2}{|c|}{$\begin{array}{c}\text { Coflex [Paradigm Spine, LLC, } \\
\text { USA] }\end{array}$} \\
\hline Allergic & $*$ \\
\hline CSF Leak & -2.25 \\
\hline Fracture & 1.33 \\
\hline Infection & -0.12 \\
\hline Malfunction & -4.95 \\
\hline Migration & 1.91 \\
\hline $\begin{array}{c}\text { Pain/Worsening } \\
\text { Symptoms }\end{array}$ & 0.75 \\
\hline
\end{tabular}
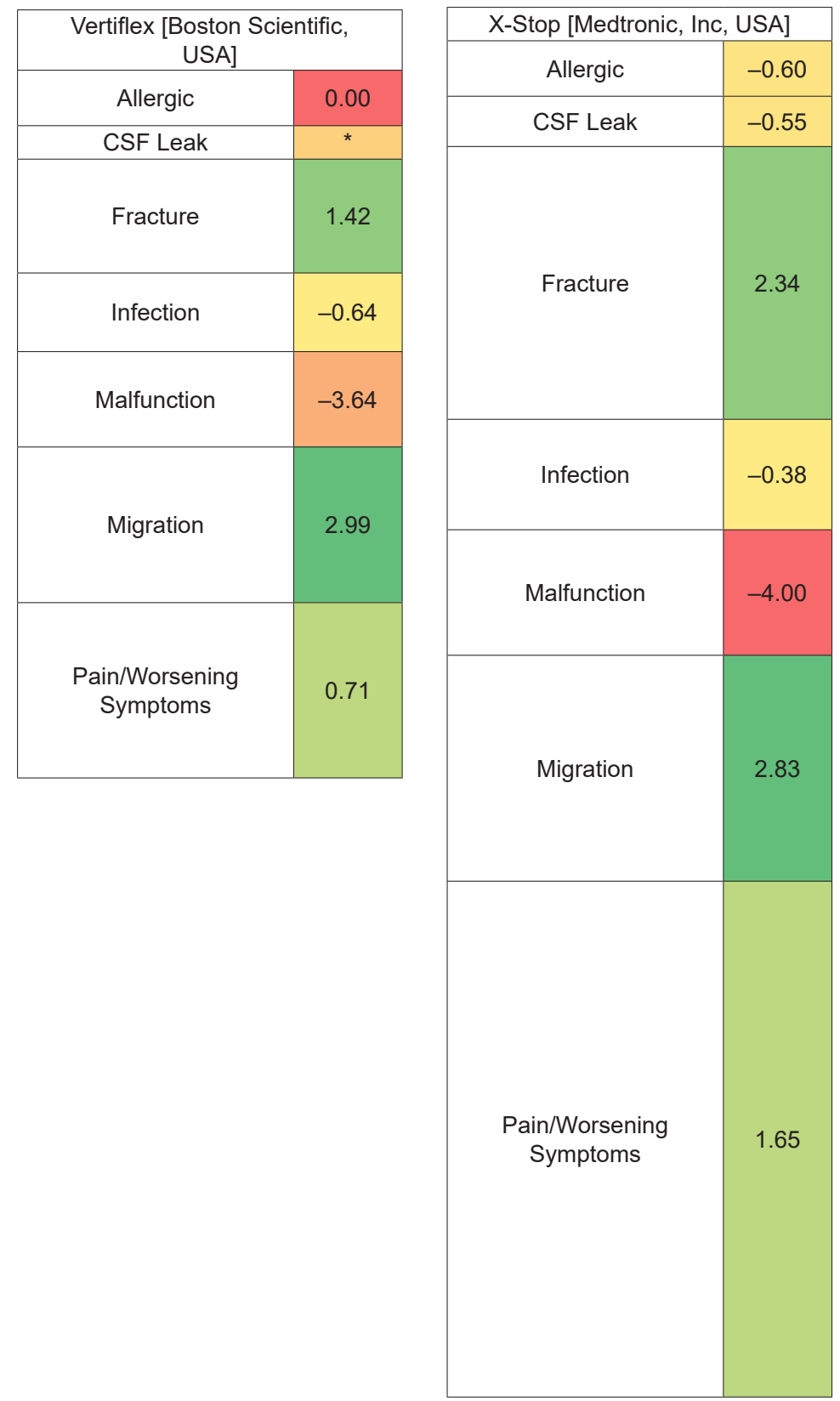

Fig. 3. Heat Map for each of the spinous process spacers/plates and adverse event combinations. $\mathrm{IC}_{025}$ for each device by adverse event is displayed in the center of the box. Color is coded by $\mathrm{IC}_{025}$ with values greater than 0 being green and those less than 0 being yellow/red. The size of each box is representative of the relative number of adverse events for that device and adverse event combination. CSF: cerebrospinal fluid, IC: information component. *These device and adverse event combinations had 0 reports and are shown with the smallest box size.

the Coflex device, including the screws. Of note, there were several cases of intervertebral disk herniation, which could cause worsening symptoms [11]. Studies have shown that the Vertiflex implant was effective in the short and longterm in relieving neurogenic claudication for lumbar spinal stenosis [12]. However, after the Vertiflex procedure, there were several patients with claudication who ended up undergoing decompressive laminectomy. In a study on Vertiflex, there were no postoperative wound infections or he- matoma noted [2], although another study identified a case of infection that occurred in an out of procedure-related adverse event from the Vertiflex implant [13]. Reviewing a randomized controlled trial, the incidence of non-healed spinous process fracture was $11.1 \%$ with Vertiflex, while healed spinous process fracture incidence was 5.3\% [14]. In the literature on X-Stop, the incidence of non-healed spinous process fracture was $5.0 \%$, while the healed spinous process fracture incidence was $3.5 \%$ [14]. Another study 
found the overall complication rate of X-Stop to be $38 \%$, including spinous process fractures and worsening symptoms [15]. In a randomized controlled trial, there was a significantly greater number of device migrations for X-Stop when compared to Vertiflex [14]. There was not much difference in the infection rate in literature between X-Stop and Vertiflex, despite the former requiring greater surgical exposure. In yet another study, no significant differences in the adverse events were found between Vertiflex and $\mathrm{X}$-Stop [16].

\section{Limitations}

The medical device reports that are submitted to the FDA and posted on the MAUDE database are submitted by health care professionals and patients. Each and every adverse event may not be reported. Selection bias exists in that only the adverse events reported are included in the analysis. The incidence or prevalence of an event cannot be determined from this database [3]. This analysis was performed on the passive surveillance system of the MAUDE database. As, such direct comparisons cannot be made between devices and adverse events signals. In addition, analyses from the database can find statistically significant signals between a device and an adverse event but cannot prove causality between them.

Randomized controlled clinical trials would be needed in order to do so. However, X-Stop has been taken off the market so randomized controlled trials for it may not be available. Analysis of the medical device reports has advantages in identifying signals in real-world situations and in diverse populations, which is near impossible with the limited number of subjects used in the randomized clinical trials [17]. Further, the heat map cannot be used to compare $\mathrm{IC}_{025}$ of one device and its adverse events with $\mathrm{IC}_{025}$ of other devices and their adverse events. It is provided as a quick reference for which device and adverse event combination contained a statistically significant signal.

In conclusion, a significant signal was found for the three interspinous spacer devices (Coflex, Vertiflex, and X-Stop) and adverse events of fracture, migration, and pain/worsening symptoms using disproportionality criteria. In addition, the heat map developed in this analysis can be used as a quick reference for determining statistically significant signals for each of the devices.

\section{CONFLICTS OF INTEREST}

No potential conflict of interest relevant to this article was reported.

\section{DATA AVAILABILITY STATEMENT}

The datasets generated during and/or analyzed during the current study are available in the The Manufacturer and User Facility Device Experience (MAUDE) database at the Food and Drug Administration.

\section{AUTHOR CONTRIBUTIONS}

Conceptualization: Nitish Aggarwal, Robert Chow. Data curation: Nitish Aggarwal. Formal analysis: Nitish Aggarwal, Robert Chow. Methodology: Nitish Aggarwal. Project administration: Nitish Aggarwal. Writing - original draft: Nitish Aggarwal. Writing - review \& editing: Robert Chow. Investigation: Robert Chow. Resources: Nitish Aggarwal. Software: Nitish Aggarwal. Supervision: Robert Chow.

\section{ORCID}

Nitish Aggarwal, https://orcid.org/0000-0003-4351-7002

Robert Chow, https://orcid.org/0000-0001-7555-0830

\section{REFERENCES}

1. Proietti L, Scaramuzzo L, Schiro' GR, Sessa S, Logroscino CA. Complications in lumbar spine surgery: a retrospective analysis. Indian J Orthop 2013; 47: 340-5.

2. Hartman J, Granville M, Jacobson RE. The use of Vertiflex ${ }^{\circ}$ interspinous spacer device in patients with lumbar spinal stenosis and concurrent medical comorbidities. Cureus 2019; 11: e5374.

3. Manufacturer and User Facility Device Experience. U.S. Food and Drug Administration [serial on the Internet]. [cited 2020 Jun 1]. Available from https://www.accessdata.fda.gov/scripts/ cdrh/cfdocs/cfMAUDE/Search.cfm? smc $=1$.

4. Tamura T, Sakaeda T, Kadoyama K, Okuno Y. Omeprazole- and esomeprazole-associated hypomagnesaemia: data mining of the public version of the FDA Adverse Event Reporting System. Int J Med Sci 2012; 9: 322-6.

5. van Puijenbroek EP, van Grootheest K, Diemont WL, Leufkens HG, Egberts AC. Determinants of signal selection in a spontaneous reporting system for adverse drug reactions. Br J Clin 
Pharmacol 2001; 52: 579-86.

6. Evans SJ, Waller PC, Davis S. Use of proportional reporting ratios (PRRs) for signal generation from spontaneous adverse drug reaction reports. Pharmacoepidemiol Drug Saf 2001; 10: 483-6.

7. Bate A, Lindquist M, Edwards IR, Olsson S, Orre R, Lansner A, et al. A Bayesian neural network method for adverse drug reaction signal generation. Eur J Clin Pharmacol 1998; 54: 315-21.

8. Belamalem S, Khadmaoui A, Tailibi I, Benkirane R, Soulaymani $\mathrm{BR}$, Soulaymani A. Use of measures of disproportionality in pharmacovigilance. Biotechnol (Rajkot) 2016; 12: 193-7.

9. Vivekanandan K, Tripathi A, Saurabh A, Kumar R, Kumar R, Prasad T, et al. Quantitative methods for the identification of signals for individual case safety reports in India. Ther Innov Regul Sci 2015; 49: 898-902.

10. Böhm R. Primer on disproportionality analysis. OpenVigil [serial on the Internet]. 2018 Oct 16 [cited 2020 Jun 1]. Available from http://openvigil.sourceforge.net/doc/DPA.pdf.

11. Mo Z, Li D, Zhang R, Chang M, Yang B, Tang S. Comparative effectiveness and safety of posterior lumbar interbody fusion, Coflex, Wallis, and X-stop for lumbar degenerative diseases: a systematic review and network meta-analysis. Clin Neurol Neurosurg 2018; 172: 74-81.
12. Deer TR, Grider JS, Pope JE, Falowski S, Lamer TJ, Calodney A, et al. The MIST Guidelines: the Lumbar Spinal Stenosis Consensus Group guidelines for minimally invasive spine treatment. Pain Pract 2019; 19: 250-74.

13. Bini W, Miller LE, Block JE. Minimally invasive treatment of moderate lumbar spinal stenosis with the superion interspinous spacer. Open Orthop J 2011; 5: 361-7.

14. Patel VV, Whang PG, Haley TR, Bradley WD, Nunley PD, Davis $\mathrm{RP}$, et al. Superion interspinous process spacer for intermittent neurogenic claudication secondary to moderate lumbar spinal stenosis: two-year results from a randomized controlled FDAIDE pivotal trial. Spine (Phila Pa 1976) 2015; 40: 275-82.

15. Bowers C, Amini A, Dailey AT, Schmidt MH. Dynamic interspinous process stabilization: review of complications associated with the X-Stop device. Neurosurg Focus 2010; 28: E8.

16. Ma X, Ma T, Dang X, Shi J, Niu N, Chang L, et al. Comparison of two interspinous spacers for treatment of moderate lumbar spinal stenosis: a meta-analysis of prospective randomized controlled trials. Int J Clin Exp Med 2017; 10: 4497-507.

17. Aggarwal P. Pyoderma gangrenosum adverse event with Rituximab use: a postmarketing pharmacovigilance analysis. Dermatol Ther 2020; 33: e13221. 